\title{
Structure, Luminescent Sensing and Proton Conduction of a Boiling-Water-Stable Zn(II) Metal-Organic Framework
}

\author{
Hua-Qun Zhou ${ }^{\dagger}$, Sai-Li Zheng ${ }^{\dagger}$, Can-Min Wu, Xin-He Ye, Wei-Ming Liao ${ }^{*}$ and Jun He *D \\ School of Chemical Engineering and Light Industry, Guangdong University of Technology, \\ Guangzhou 510006, China; 3114001689@mail2.gdut.edu.cn (H.-Q.Z.); 1111906003@mail2.gdut.edu.cn (S.-L.Z.); \\ 3217003865@mail2.gdut.edu.cn (C.-M.W.); 2111906026@mail2.gdut.edu.cn (X.-H.Y.) \\ * Correspondence: wmliao@gdut.edu.cn (W.-M.L.); junhe@gdut.edu.cn (J.H.) \\ + These authors contributed equally to this work.
}

Citation: Zhou, H.-Q.; Zheng, S.-L.; Wu, C.-M.; Ye, X.-H.; Liao, W.-M.; He, J. Structure, Luminescent Sensing and Proton Conduction of a Boiling-Water-Stable Zn(II) Metal-Organic Framework. Molecules 2021, 26, 5044. https://doi.org/ $10.3390 /$ molecules 26165044

Academic Editor: Anna Cleta Croce

Received: 23 July 2021

Accepted: 17 August 2021

Published: 20 August 2021

Publisher's Note: MDPI stays neutral with regard to jurisdictional claims in published maps and institutional affiliations.

Copyright: (c) 2021 by the authors. Licensee MDPI, Basel, Switzerland. This article is an open access article distributed under the terms and conditions of the Creative Commons Attribution (CC BY) license (https:/ / creativecommons.org/licenses/by/ $4.0 /)$.

\begin{abstract}
A novel Zn(II) metal-organic framework $\left[\mathrm{Zn}_{4} \mathrm{O}\left(\mathrm{C}_{30} \mathrm{H}_{12} \mathrm{~F}_{4} \mathrm{O}_{4} \mathrm{~S}_{8}\right)_{3}\right]_{n}$, namely ZnBPD-4F4TS, has been constructed from a fluoro- and thiophenethio-functionalized ligand 2,2',5,5'-tetrafluoro3,3',6,6'-tetrakis(2-thiophenethio)-4,4'-biphenyl dicarboxylic acid ( $\mathbf{H}_{2}$ BPD-4F4TS). ZnBPD-4F4TS shows a broad green emission around $520 \mathrm{~nm}$ in solid state luminescence, with a Commission International De L'Eclairage (CIE) coordinate at $\mathrm{x}=0.264, \mathrm{y}=0.403$. Since $\mathrm{d}^{10}$-configured $\mathrm{Zn}$ (II) is electrochemically inert, its photoluminescence is likely ascribed to ligand-based luminescence which originates from the well-conjugated system of phenyl and thiophenethio moieties. Its luminescent intensities diminish to different extents when exposed to various metal ions, indicating its potential as an optical sensor for detecting metal ion species. Furthermore, ZnBPD-4F4TS and its $\mathrm{NH}_{4}$ Br-loaded composite, $\mathbf{N H}_{4}$ Br@ZnBPD-4F4TS, were used for proton conduction measurements in different relative humidity $(\mathrm{RH})$ levels and temperatures. Original ZnBPD-4F4TS shows a low proton conductivity of $9.47 \times 10^{-10} \mathrm{~S} \mathrm{~cm}^{-1}$ while $\mathbf{N H}_{4} \mathbf{B r} @ Z \mathbf{Z n B D}-4 \mathrm{~F} 4 \mathrm{TS}$ shows a more than 25,000 -fold enhanced value of $2.38 \times 10^{-5} \mathrm{~S} \mathrm{~cm}^{-1}$ at $40{ }^{\circ} \mathrm{C}$ and $90 \% \mathrm{RH}$. Both of the proton transport processes in ZnBPD-4F4TS and $\mathbf{N H}_{4}$ Br@ZnBPD-4F4TS belong to the Grotthuss mechanism with $E_{\mathrm{a}}=0.40$ and $0.32 \mathrm{eV}$, respectively.
\end{abstract}

Keywords: proton conduction; metal ion sensing; luminescence; metal-organic framework

\section{Introduction}

The luminescent metal-organic framework (LMOF) is a fascinating class of functional materials that has been extensively researched for its inspiring application in plentiful areas, especially chemical sensing of molecules and cation and anion species [1-4]. The luminescent and sensing property of LMOFs is related to original metal ions, functional ligands and self-assembly processes. To date, LMOF-based sensors have mainly focused on lanthanide-based (e.g., $\mathrm{Eu}^{3+}, \mathrm{Tb}^{3+}$ ) metal-organic frameworks largely because of their strong photoluminescence derived from the ligand-to-lanthanide antenna effect [5-8]. The high cost and almost unalterable emission wavelength of lanthanide LMOFs motivate researchers to develop LMOFs using transition metal ions (e.g., $\mathrm{Zn}^{2+}, \mathrm{Cd}^{2+}$, etc.) as metal nodes [9-11]. As some $\mathrm{d}^{10}$ metal ions (e.g., $\mathrm{Zn}^{2+}$ ) are proposed to exert less influence on the emission of LMOFs, ligand design is important for designing Zn-LMOF phosphors [12-14]. The introduction of an aromatic $\pi$-conjugated system into a ligand helps promote the red shift of the emission wavelength to the visible light region and improves the emission intensity. Further, aromatic groups are proposed to boost the interaction between the host framework and substrate $[15,16]$. Metal ion detection has been focused on extensively because excess use and emission of metal species have caused many issues, such as environmental pollution, health hazards, etc. $[17,18]$. Nevertheless, it is still a challenge to achieve LMOF-based sensors with high selectivity, sensitivity and recyclability. In particular, only a few examples of LMOF-based sensors of $\mathrm{Mn}^{2+}$ ions have been reported $[19,20]$. 
In recent years, MOF-based proton-conducting materials have received much attention due to their potential application in proton exchange membrane fuel cells [21-24]. Traditional Nafion materials have the shortcomings of high cost and intolerance of high temperature, which also accelerates the development of candidate materials [25]. MOFs can be constructed by commonly available metal ions and well-designed ligands, and many of them exhibit rather high solvent (i.e., $\mathrm{H}_{2} \mathrm{O}$ ) and thermal (over $400{ }^{\circ} \mathrm{C}$ ) stability [26-28]. This feature makes them an advantage in water-assisted proton conducting materials. Hydrated proton conduction is mainly determined by a hydrogen bond network created by appropriate porosity, functional groups on the ligand and proton carriers in the voids. This leads to two kinds of strategies for achieving high proton conductivity: (1) introducing acidic and hydrophilic groups including F, N, O and S atoms to the ligand [29-33]; (2) encapsulating guest molecules (e.g., water, imidazole, histamine, ammonium bromide, etc.) into the voids [34-38].

Herein, thiophenethio- and fluorine-functionalized linker molecule 2,2',5,5'-tetrafluoro-

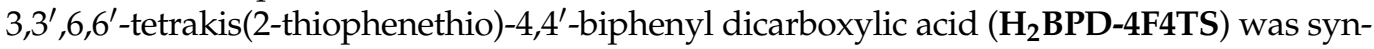
thesized and used to react with $\mathrm{Zn}\left(\mathrm{NO}_{3}\right)_{2}$ through a solvothermal strategy, affording a metalorganic framework, ZnBPD-4F4TS. Photoluminescent measurement and metal ion sensing experiments for ZnBPD-4F4TS have been performed, showing metal ion-dependent quenching with luminescence intensity. The existence of pendant $\mathrm{F}$ - and S-included groups provide a platform for adjusting proton conduction. The $\mathrm{NH}_{4} \mathrm{Br}$-loaded composite, $\mathbf{N H}_{4} \mathbf{B r} @ \mathbf{Z n B P D}$ 4F4TS, exhibits a highly improved conductivity $\left(2.38 \times 10^{-5} \mathrm{~S} \mathrm{~cm}^{-1}\right)$ compared to that of original ZnBPD-4F4TS $\left(9.47 \times 10^{-10} \mathrm{~S} \mathrm{~cm}^{-1}\right)$. It highlights encapsulating proton carriers into the framework as an effective strategy to enhance proton transport.

\section{Results and Discussion}

\subsection{Synthesis and Structure Characterization}

Ligand $\mathbf{H}_{2}$ BPD-4F4TS, 2,2',5,5' -tetrafluoro-3,3',6,6' ${ }^{\prime}$-tetrakis(2-thiophenethio)-4,4'-biphenyl dicarboxylic acid, was synthesized according to our previous work [39]. Further selfassembly with zinc nitrate hexahydrate afforded light yellow square crystals (Figure S1), namely ZnBPD-4F4TS. The rigid framework of ZnBPD-4F4TS was well determined by single crystal X-ray diffraction (SCXRD) analysis (Figure 1). Unfortunately, the thiophene substituents were highly disordered and difficult to resolve. Therefore, ZnBPD-4F4TS was digested (in $\mathrm{DCl} / \mathrm{NaF} / \mathrm{DMSO}-d_{6}$ ) for ${ }^{1} \mathrm{H}$ and ${ }^{19} \mathrm{~F}$ NMR tests to verify the intact thiophene units of linker BPD-4F4TS ${ }^{2-}$ (Figures S2 and S3).

SCXRD results reveals that ZnBPD-4F4TS crystallizes in the cubic $I 23$ space group with a $=17.232 \AA$ and $\alpha=90^{\circ}$ (Table S1). The asymmetric unit is built from one crystallographically independent $\mathrm{Zn}^{2+}$ ion, one oxygen and 1/4 BPD-4F4TS ${ }^{2-}$ anion. The metal node consists of a $\mathrm{Zn}-\mathrm{O}$ cluster-based secondary building unit (SBU) with a formula of $\mathrm{Zn}_{4} \mathrm{O}(\mathrm{COO})_{6}$ (Figure 1a). Each of these four $\mathrm{Zn}^{2+}$ ions is located at a tetrahedron center (cyan tetrahedron represents the $\mathrm{ZnO}_{4}$ polyhedron) and bridged by one $\mu_{4}-\mathrm{O}$. Each $\mathrm{Zn}^{2+}$ ion bonds to four oxygen atoms in which one comes from a $\mu_{4}-\mathrm{O}$ and the other three are from three carboxylate groups of BPD-4F4TS ${ }^{2-}$. Each linear BPD-4F4TS ${ }^{2-}$ (Figure 1b) acts as a two-connected bridge to link the SBUs, forming a 3D framework (Figure 1c). Interconnected 1D channels partly filled by thiophene groups run along the $a, b$ and $c$ crystallographic axes (Figure 1d). The coordination environment of the $\mathrm{Zn}^{2+}$ ion and coordination mode of BPD4F4TS ${ }^{2-}$ in ZnBPD-4F4TS are the same as previously reported IRMOF-type MOFs [40], but they show a two-fold interpenetrated aggregation (Figure 1d). The construction of SBUs and linkers can be simply reticulated into a pcu topology (Figure 1e).

Pure phase of as-synthesized ZnBPD-4F4TS was obtained with the solvothermal method according to a consistent powder X-ray diffraction (SCXRD) pattern with the simulated one, which was also verified by FT-IR spectra (Figure 2 and Figure S4). $\mathbf{N H}_{4} \mathbf{B r} @ \mathbf{Z n B P D}-4 \mathrm{~F} 4 \mathrm{TS}$ was prepared by immersing ZnBPD-4F4TS in a saturated $\mathrm{NH}_{4} \mathrm{Br}$ solution of ethanol at room temperature for 2 days. The loading amount of $\mathrm{NH}_{4} \mathrm{Br}$ in the voids was further determined to be $2.21 \mathrm{wt} \%$ by elemental analysis (EA) measurement. 
(a)

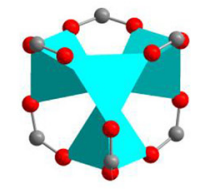

(b)

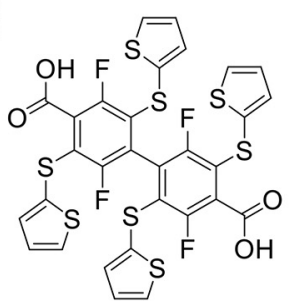

(e)

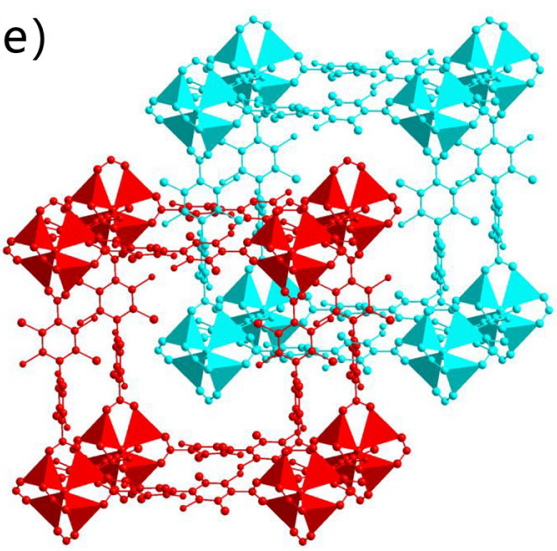

(c)

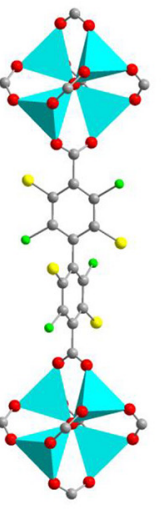

(d)

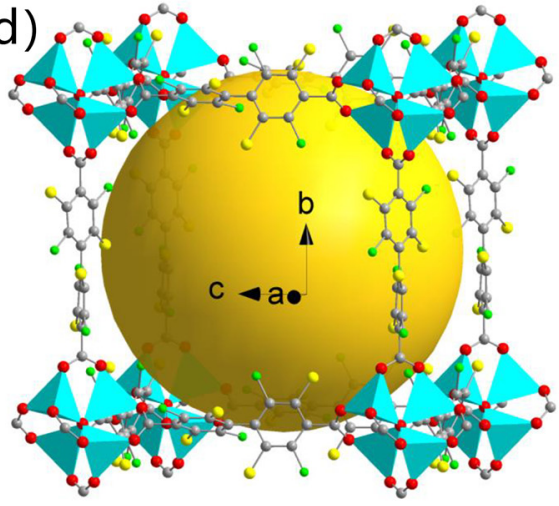

(f)

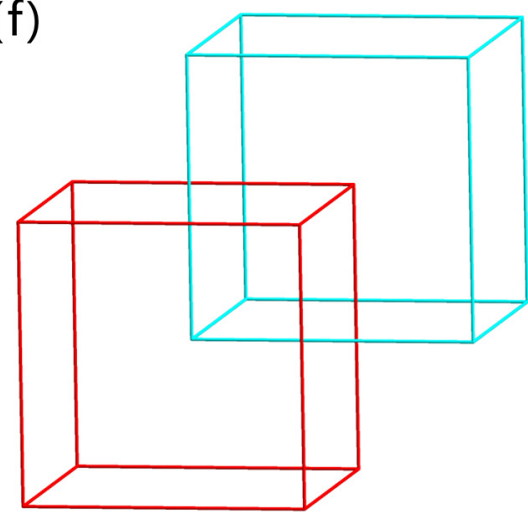

Figure 1. (a) $\mathrm{Zn}_{4} \mathrm{O}(\mathrm{COO})_{6}$ cluster of ZnBPD-4F4TS. (b) Ligand $\mathbf{H}_{2}$ BPD-4F4TS used in this work. (c) Coordination mode diagram of linker BPD-4F4TS ${ }^{2-}$. (d) A three-dimensional network diagram of ZnBPD-4F4TS. The ball is present to display the inner void filled with disordered pendent thiophene groups. (e) Two-fold interpenetrated framework of ZnBPD-4F4TS. (f) A simplified topology of ZnBPD-4F4TS. Hydrogen atoms are omitted for clarity and thiophene groups are absent because of their high disorder. Atom color: red, oxygen; gray, carbon; green, fluorine; yellow, sulfur; cyan, $\mathrm{Zn}_{4} \mathrm{O}$ polyhedron.

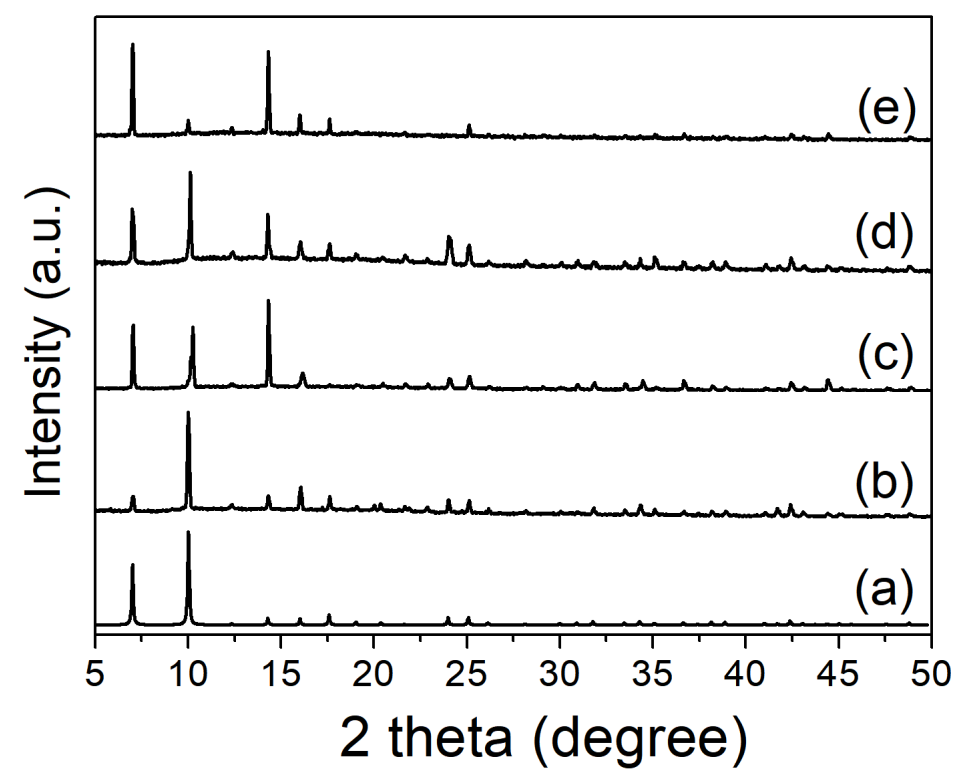

Figure 2. PXRD patterns of ZnBPD-4F4TS: (a) A simulated one obtained from single crystal data, (b) as-synthesized one, (c) after exposed to air for 21 days, (d) activated sample after acetone exchange and thermal treatment at $100{ }^{\circ} \mathrm{C}$ and vacuum, (e) after soaking in boiling water for $30 \mathrm{~h}$. 


\subsection{Stability of ZnBPD-4F4TS}

The stability of metal-organic frameworks is vital to their practical applications; therefore, the atmospheric, thermal and solvent stabilities have been investigated. ZnBPD4F4TS was highly air and water stable judging from the unchanged PXRD patterns after exposure to the atmosphere for 21 days and soaking in boiling water for $30 \mathrm{~h}$, respectively (Figure 2(c,e)). This excellent stability of ZnBPD-4F4TS to water is rare in reported Zn(II)carboxylate frameworks [26,41]. As-synthesized crystalline ZnBPD-4F4TS was further Soxhlet-extracted by acetone and heated at $100{ }^{\circ} \mathrm{C}$ to prepare activated ZnBPD-4F4TS. EA data indicated a formula of $\left[\mathrm{Zn}_{4} \mathrm{O}\left(\mathrm{C}_{30} \mathrm{H}_{12} \mathrm{~F}_{4} \mathrm{O}_{4} \mathrm{~S}_{8}\right)_{3} \cdot\left(\mathrm{H}_{2} \mathrm{O}\right)_{1.5}\left(\mathrm{CH}_{3} \mathrm{CN}\right)_{0.6}\right]_{n}$ for activated ZnBPD-4F4TS. Consistent diffraction peaks with as-synthesized ZnBPD-4F4TS suggested an intact coordination framework (Figure 2(d)). The thermogravimetric (TG) curve of activated ZnBPD-4F4TS showed slight weight loss (3.15\%) in the initial period, which could be attributed to the loss of a few water molecules from the air and remaining crystallized guest molecules in the pores. This result suggested the coordination framework was thermally stable below $380^{\circ} \mathrm{C}$ (Figure S5). The subsequent large weight loss could be ascribed to decomposition of organic ligands and destruction of coordination bonds, which was supported by the endothermic effect in the differential thermal analysis (DTA) curve (Figure S5). The consistent PXRD pattern of $\mathrm{NH}_{4} \mathrm{Br} @ \mathrm{ZnBPD}-4 \mathrm{~F} 4 \mathrm{TS}$ with ZnBPD-4F4TS indicated that the host framework remained stable after immersion in $\mathrm{NH}_{4} \mathrm{Br}$ solution (Figure S6b).

\subsection{Luminescent Properties}

With the conjugated thiophene and phenyl units, ZnBPD-4F4TS was expected to possess decent luminescent property. Both excitation and emission spectra of ligand $\mathbf{H}_{\mathbf{2}}$ BPD-4F4TS and ZnBPD-4F4TS were recorded in the solid state (Figure 3a,b). In the case of ligand $\mathbf{H}_{2}$ BPD-4F4TS, it displays one fluorescent emission band centered at $535 \mathrm{~nm}$ when excited at $370 \mathrm{~nm}$, which is probably assigned to $\pi$ or $n$ to $\pi^{*}$ orbital transitions $[42,43]$. After coordination to form ZnBPD-4F4TS, it shows a similar but blue-shifted emission peak at $518 \mathrm{~nm}$ when excited at $370 \mathrm{~nm}$, showing a green emission of crystals (Figure 3c). This blue shift of ZnBPD-4F4TS in comparison to free ligand is probably attributed to the metal-ligand coordination interaction and deprotonated effect of the dicarboxylic acid [44,45]. Accordingly, Commission International De L'Éclairage (CIE) coordinates change from $(0.297,0.396)$ for the ligand to $(0.264,0.403)$ for ZnBPD-4F4TS (Figure 3d). In addition, the emission spectrum of $\mathbf{N H}_{4}$ Br@ZnBPD-4F4TS was also obtained and found to be quite similar to that of as-synthesized ZnBPD-4F4TS (Figure S7).

\subsection{Metal Sensing and Mechanism}

Excellent water stability, free-standing thiophenethio-functions in the pores and potential accessible pores inspired us to investigate the chemical sensing performance of ZnBPD-4F4TS in aqueous media. As-synthesized crystals of ZnBPD-4F4TS were first immersed in $500 \mathrm{ppm}$ (based on metal species) metal chloride or nitrate solutions $\left(\mathrm{Pb}^{2+}\right.$, $\mathrm{Pd}^{2+}, \mathrm{Co}^{2+}, \mathrm{Fe}^{3+}, \mathrm{Ni}^{2+}, \mathrm{Hg}^{2+}, \mathrm{Cu}^{2+}, \mathrm{Cd}^{2+}, \mathrm{Pt}^{2+}, \mathrm{Mn}^{2+}$ and $\left.\mathrm{Ag}^{+}\right)$for $2 \mathrm{~h}$ at $80{ }^{\circ} \mathrm{C}$ and then selected for photography (Figure 4a). All of these crystals show no obvious color change, though some exhibit a semi-transparent appearance. When irradiated under $365 \mathrm{~nm}$ UV light, these crystals exhibit similar emission colors but with variously decreased brightness in comparison to as-synthesized ZnBPD-4F4TS. Emission spectra of bulk samples were also measured in the wavelength range of 380 to $720 \mathrm{~nm}$, and they show different extents of reduction in luminescence intensity (monitored at $518 \mathrm{~nm}$ ) after immersion in various metal ion solutions (Figure $4 \mathrm{~b}$ ). In particular, the case of $\mathrm{Mn}^{2+}$ exhibits the strongest luminescence quenching (Figure 4c). PXRD patterns of ZnBPD-4F4TS were found to be unchanged after exposure to various metal ion solutions, suggesting an intact framework structure (Figure S8). 

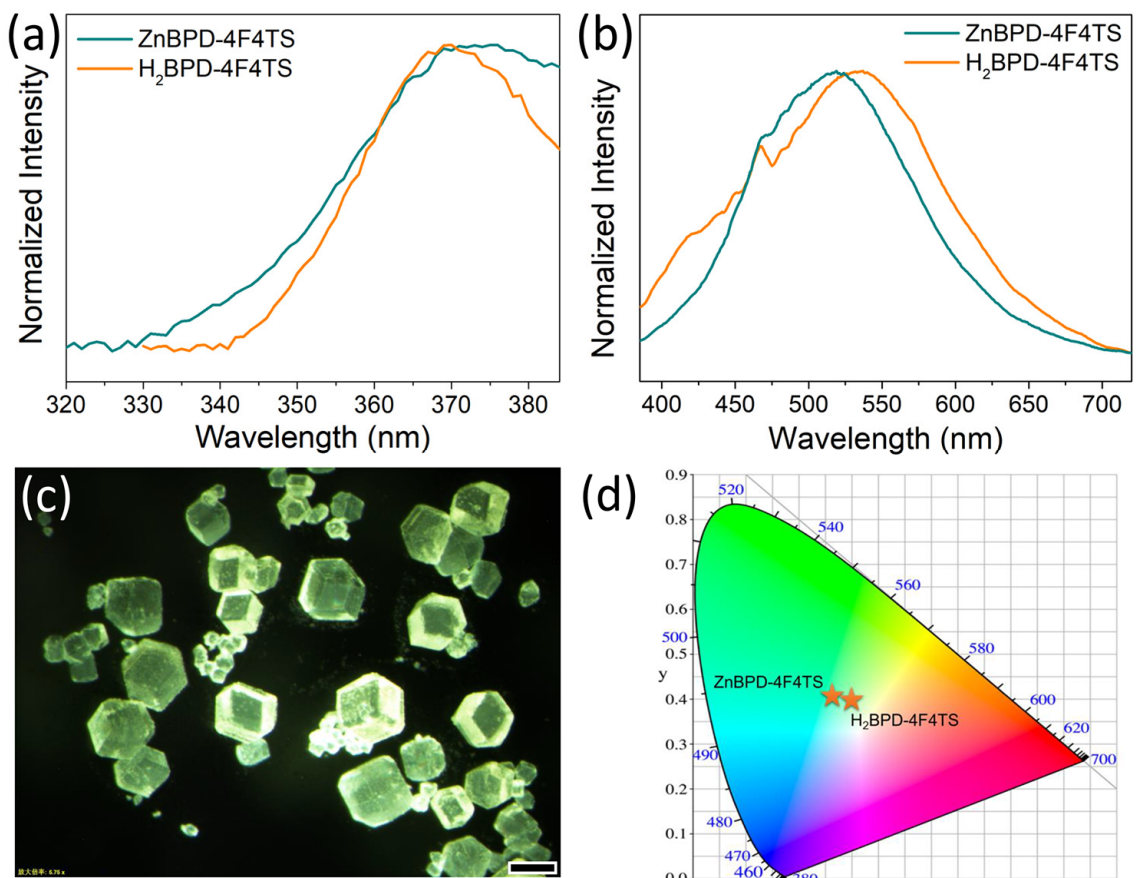

(d)

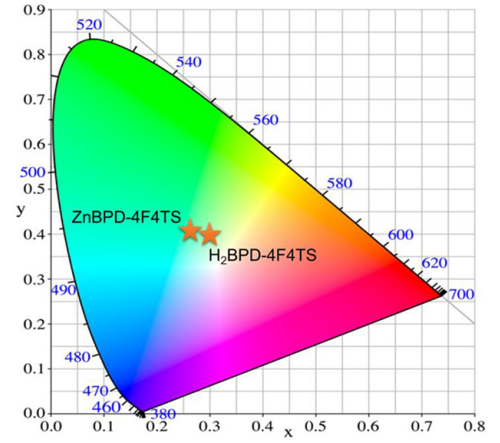

Figure 3. (a) Excitation spectra of ligand $\mathbf{H}_{2}$ BPD-4F4TS $\left(\lambda_{\mathrm{em}}=535 \mathrm{~nm}\right)$ and ZnBPD-4F4TS $\left(\lambda_{\mathrm{em}}=518 \mathrm{~nm}\right)$ in the solid state. (b) Emission spectra of ligand $\mathbf{H}_{\mathbf{2}}$ BPD-4F4TS and ZnBPD-4F4TS when excited at $370 \mathrm{~nm}$ UV light in the solid state. (c) A photograph of as-synthesized ZnBPD-4F4TS single crystals under UV light irradiation. The scale in the figure is $100 \mu \mathrm{m}$. (d) CIE chromaticity diagram of ligand $\mathbf{H}_{2}$ BPD-4F4TS and ZnBPD-4F4TS when excited at $370 \mathrm{~nm}$.

(a)

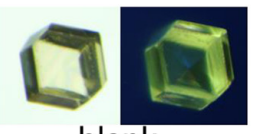

blank
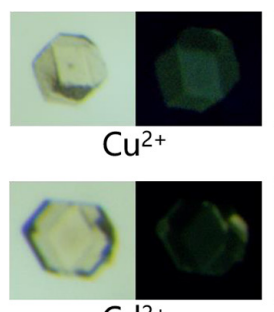

$\mathrm{Cd}^{2+}$

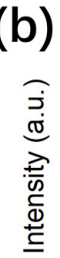

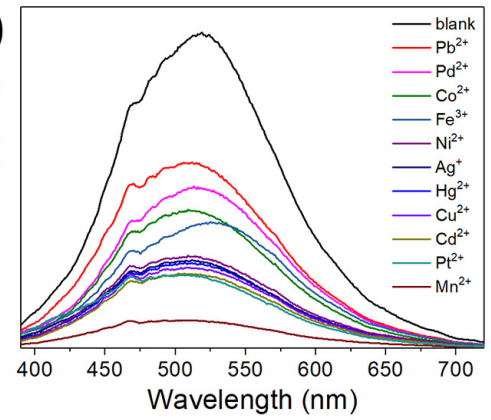

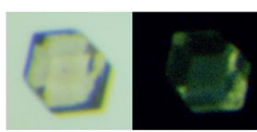

$\mathrm{Ag}^{+}$

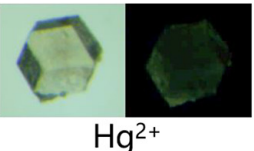

$\mathrm{Hg}^{2+}$

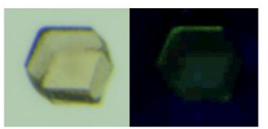

$\mathrm{Pb}^{2+}$

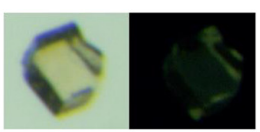

$\mathrm{Ni}^{2+}$

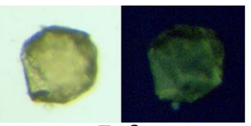

$\mathrm{Fe}^{3+}$

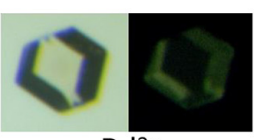

$\mathrm{Pd}^{2+}$

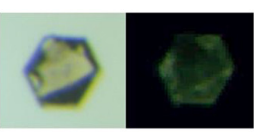

$\mathrm{Co}^{2+}$

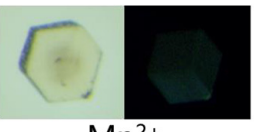

$\mathrm{Mn}^{2+}$

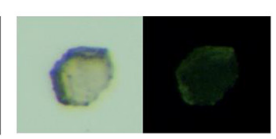

$\mathrm{Pt}^{2+}$

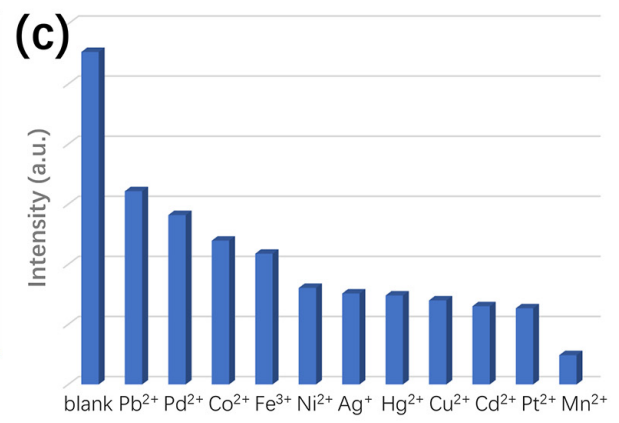

Figure 4. (a) Photographs of ZnBPD-4F4TS crystals under natural light and $365 \mathrm{~nm}$ UV light irradiation after immersion in water and aqueous solution of different metal ions (500 ppm). (b) Room temperature emission spectra $\left(\lambda_{\mathrm{ex}}=370 \mathrm{~nm}\right)$ of ZnBPD-4F4TS after immersion in water and aqueous solution of different metal ions (500 ppm). (c) Emission intensity data at $518 \mathrm{~nm}$ according to the spectra from $(\mathbf{b})$. 


\subsection{Proton Conduction}

The proton carrier is one of the vital factors for improving proton conductivity. This motivated us to optimize the as-synthesized framework by encapsulating protonic guests (i.e., $\mathrm{NH}_{4} \mathrm{Br}$ ) into framework voids through a post-synthetic strategy. After ZnBPD4F4TS was immersed in $\mathrm{NH}_{4} \mathrm{Br} / \mathrm{EtOH}$ solution for 2 days, $\mathrm{NH}_{4} \mathbf{B r} \mathrm{ZnBPD}-4 \mathrm{~F} 4 \mathrm{TS}$ was separated, washed and dried. The ac impedance measurements were carried out with compacted pellets of ZnBPD-4F4TS and $\mathbf{N H}_{4} \mathrm{Br} @ Z n B P D-4 F 4 T S$, respectively. The corresponding Nyquist plots are shown at different temperatures at $90 \%$ relative humidity $(\mathrm{RH})$ (Figure $5 \mathrm{a}, \mathrm{c}$ ). In a typical measurement, the proton conductivity of a sample is determined by the high frequency region with the following equation:

$$
\sigma=l / R S
$$

where $l$ is the thickness $(\mathrm{mm})$ and $S$ is the cross-sectional area $\left(\mathrm{mm}^{2}\right)$ of the pellet, while $R(\Omega)$ can be calculated from the Nyquist impedance plots $[46,47]$. Accordingly, the corresponding proton conductivity was obtained and is listed in Table S2. It is exciting that $\mathbf{N H}_{4} \mathbf{B r} @ Z n B P D-4 F 4 T S\left(2.38 \times 10^{-5} \mathrm{~S} \cdot \mathrm{cm}^{-1}\right)$ exhibits a 25,000-fold increased proton conductivity compared to the original ZnBPD-4F4TS $\left(9.47 \times 10^{-10} \mathrm{~S} \cdot \mathrm{cm}^{-1}\right)$ at $40^{\circ} \mathrm{C}$ and $90 \%$ RH. This high and sharply increased proton conductivity of $\mathbf{N H}_{4} \mathbf{B r} @ Z n B P D-$ 4F4TS highlights that manipulation of protonic guests serves as an effective strategy to promote proton transport in the framework. Further research reveals that ZnBPD4F4TS possesses increasing proton conductivity varying from $9.47 \times 10^{-10}\left(40^{\circ} \mathrm{C}\right)$ to $4.19 \times 10^{-9}\left(80^{\circ} \mathrm{C}\right) \mathrm{S} \cdot \mathrm{cm}^{-1}$ when the temperature increases under $90 \% \mathrm{RH}$ (Figure S9). Similarly, the conductivity of $\mathbf{N H}_{4} \mathbf{B r} @ Z n B P D-4 F 4 T S$ increases from to $2.38 \times 10^{-5}\left(40^{\circ} \mathrm{C}\right)$ to $7.87 \times 10^{-5}\left(80^{\circ} \mathrm{C}\right) \mathrm{S} \cdot \mathrm{cm}^{-1}$ (Figure S9). Both of the metal-organic frameworks remain stable after proton conduction measurements according to the unchanged PXRD patterns (Figures S6 and S10).
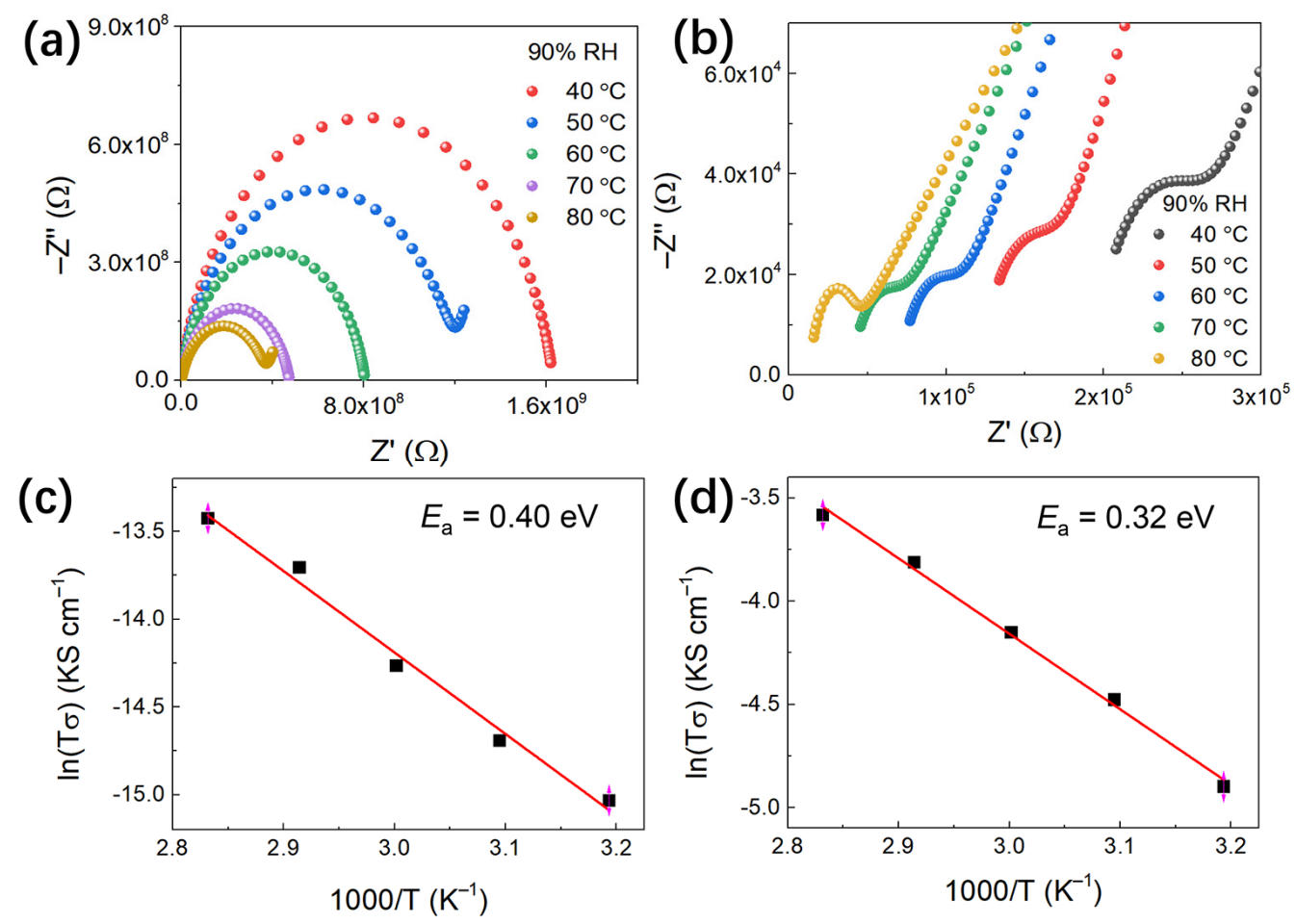

Figure 5. Nyquist plots of (a) ZnBPD-4F4TS and (b) $\mathrm{NH}_{4} \mathrm{Br} @ Z n B P D-4 F 4 T S$ at different temperatures (from 40 to $80{ }^{\circ} \mathrm{C}$ ) and $90 \%$ RH. Dependence of proton conductivity in (c) ZnBPD-4F4TS and (d) $\mathbf{N H}_{4}$ Br@ZnBPD-4F4TS as a function of temperature at $90 \% \mathrm{RH}$. 


\subsection{Proton Conduction Mechanism}

The obtained conductivities of ZnBPD-4F4TS and $\mathbf{N H}_{4}$ Br@ZnBPD-4F4TS at various temperatures are plotted against temperature in the form of $\ln (\sigma T)$ against 1000/T (Figure $5 b, d$ ), and the activation energy $\left(E_{\mathrm{a}}\right)$ of proton transport can be estimated using the Arrhenius equation:

$$
\sigma T=\sigma_{0} \exp \left(-E_{a} / k T\right)
$$

The $E_{a}$ value is calculated to be 0.40 and $0.32 \mathrm{eV}$ for ZnBPD-4F4TS and $\mathbf{N H}_{4} \mathbf{B r} @ Z \mathbf{Z n B D}$ 4F4TS, respectively. Generally, the proton transport process is classified into Grotthuss [48] $\left(E_{a}<0.4 \mathrm{eV}\right)$ and vehicle [49] $\left(E_{a}>0.4 \mathrm{eV}\right)$ mechanisms according to the $E_{a}$ value. Therefore, both of these materials can be attributed to the Grotthuss mechanism. It indicates that proton transport is achievable through the hydrogen bond network of proton carriers. Both of the $\mathrm{H}_{2} \mathrm{O}$ molecules and electronegative $\mathrm{S}$ atoms in the framework help form the hydrogen bonds to promote proton transport. $\mathbf{N H}_{4} \mathbf{B r} @ \mathbf{Z n B P D}-4 \mathbf{F} 4 \mathbf{T S}$ shows a 25,000-fold improvement in conductivity compared to ZnBPD-4F4TS that might originate from the more favorable hydrogen bond channels among $\mathrm{H}_{2} \mathrm{O}$, thiophene and $\mathrm{NH}_{4} \mathrm{Br}$ species.

\section{Materials and Methods}

\subsection{General Procedure}

Starting materials, reagents and solvents were purchased from commercial sources (J\&K, Aldrich and Acros) and used without further purification. Elemental analysis (EA) was performed with a Vario Micro CUBE CHN elemental analyzer (Elementar, Germany). FT-IR spectra were obtained using a Avatar 360 spectrophotometer (Thermo Nicolet, The United States). Nuclear magnetic resonance (NMR) spectra were recorded at $298 \mathrm{~K}$ on a $400 \mathrm{MHz}$ superconducting magnet high-field NMR spectrometer (Bruker, The Swiss), with working frequencies of $400 \mathrm{MHz}$ for ${ }^{1} \mathrm{H}, 376 \mathrm{MHz}$ for ${ }^{19} \mathrm{~F}$. Chemical shifts are reported in ppm relative to the signals corresponding to the residual non-deuterated solvents, with tetramethylsilane (TMS) as the internal standard. Thermogravimetric (TG) analyses were carried out in a nitrogen stream using Thermal analysis equipment (STA 6000) (PerkinElmer, The United States) with a heating rate of $10^{\circ} \mathrm{C} / \mathrm{min}$. Powder X-ray diffraction (PXRD) data were collected in reflection mode at room temperature on a Smart Lab diffractometer (Rigaku, Japan) with a mixture of $\mathrm{Cu}-\mathrm{K} \alpha 1(\lambda=1.54056 \AA)$ and $\mathrm{Cu}-\mathrm{K} \alpha 2(\lambda=1.5418 \AA)$ radiation. Fluorescence spectra were measured on a FluoroMax-4 fluorescence spectrometer (HORIBA Jobin Yvon, France) at room temperature.

\subsection{Synthesis of $\left[\mathrm{Zn}_{4} \mathrm{O}\left(\mathrm{H}_{2} \mathrm{BPD}-4 \mathrm{~F} 4 \mathrm{TS}\right)_{3}\right]_{n}$ (ZnBPD-4F4TS)}

The ligand $\mathbf{H}_{2}$ BPD-4F4TS (5 mg, $0.0065 \mathrm{mmol}$ ) and zinc nitrate hexahydrate (5 mg, $0.017 \mathrm{mmol}$ ) were weighed into a glass tube $8 \mathrm{~mm}$ in diameter, and then a mixed solvent of water and acetonitrile $(0.8 \mathrm{~mL}, v / v=1: 1)$ was added. The mixture was sonicated for $5 \mathrm{~min}$ to form a clear solution. Then, the glass tube nozzle was melted and sealed at high temperature, and the glass tube was heated at $140{ }^{\circ} \mathrm{C}$ for $36 \mathrm{~h}$, followed by natural cooling to room temperature, during which light yellow truncated cube-like crystals $(2.4 \mathrm{mg}, 14 \%$ based on $\mathbf{H}_{2}$ BPD-4F4TS) were formed.

\subsection{Activation of ZnBPD-4F4TS}

To exchange and remove the solvent molecules from the pores of ZnBPD-4F4TS, a thimble (e.g., made from folding filter paper) containing as-synthesized ZnBPD-4F4TS crystals $(50 \mathrm{mg})$ was loaded into the main chamber of a Soxhlet extractor. The Soxhlet extractor was connected to a $250 \mathrm{~mL}$ round-bottomed flask including acetone $(150 \mathrm{~mL})$ and a magnetic stirring bar, and then equipped with a water condenser. The flask was heated to $100{ }^{\circ} \mathrm{C}$ with an oil bath for 3 days. The filter paper was then taken out and the solid was heated at $90{ }^{\circ} \mathrm{C}$ under vacuum to give the activated ZnBPD-4F4TS sample. Elemental analysis found [C (41.35\%), H (1.57\%), S (29.81\%), N (0.33\%)], a fitting formula can be determined to be $\mathrm{Zn}_{4} \mathrm{O}\left(\mathrm{C}_{30} \mathrm{H}_{12} \mathrm{~F}_{4} \mathrm{O}_{4} \mathrm{~S}_{8}\right)_{3}\left(\mathrm{H}_{2} \mathrm{O}\right)_{1.5}\left(\mathrm{CH}_{3} \mathrm{CN}\right)_{0.6}$ (m.w. 2635.86), which gives a calculated profile as [C (41.56\%), H $(1.56 \%), \mathrm{S}(29.19 \%), \mathrm{N}(0.32 \%)$ ]. FT-IR (KBr 
pellet, v/ $\left.\mathrm{cm}^{-1}\right)$ : $3444(\mathrm{w}), 1622$ (s), 1434 (s), 1398 (s), 1385 (s), 1363 (s), 1218 (m), 1143 (w), $1103(\mathrm{w}), 986(\mathrm{~m}), 907(\mathrm{~m}), 850(\mathrm{~m}), 803(\mathrm{~m}), 767(\mathrm{w}), 741(\mathrm{~m}), 698(\mathrm{~s}), 623(\mathrm{w}), 584(\mathrm{w})$, $517(\mathrm{w}), 477(\mathrm{w}), 447(\mathrm{w})$.

\subsection{Synthesis of $\mathrm{NH}_{4} \mathrm{Br} @ \mathrm{ZnBPD-4F4TS}$}

The solid sample of as-synthesized ZnBPD-4F4TS (30 mg) was added to a small glass bottle containing $4 \mathrm{~mL}$ of saturated ammonium bromide in ethanol solution, and soaked at room temperature for 2 days. Afterwards, the resultant solid $\mathbf{N H}_{4} \mathbf{B r} @ Z$ ZnBPD-4F4TS was isolated by centrifugation and then washed with ethanol and acetone three times, and dried under vacuum for $1 \mathrm{~h}$. Elemental analysis found [C (40.99\%), H (1.52\%), S (28.66\%), N $(0.37 \%)]$, a fitting formula can be determined to be $\mathrm{Zn}_{4} \mathrm{O}\left(\mathrm{C}_{30} \mathrm{H}_{12} \mathrm{~F}_{4} \mathrm{O}_{4} \mathrm{~S}_{8}\right)_{3}\left(\mathrm{H}_{2} \mathrm{O}\right)_{0.5}\left(\mathrm{NH}_{4} \mathrm{Br}\right)_{0.6}$ $\left(\mathrm{CH}_{3} \mathrm{CN}\right)_{0.1}$ (m.w. 2656.08), which gives a calculated profile as [C (40.79\%), H (1.51\%), S $(28.97 \%), \mathrm{N}(0.37 \%)]$.

\subsection{Single Crystal X-ray Crystallography}

Single crystal data for ZnBPD-4F4TS were collected using a Bruker APEX-II CCD diffractometer (Bruker, Germany) with an I-mu-S micro-focus X-ray source using $\mathrm{Cu} \mathrm{K} \alpha$ radiation $(\lambda=1.54178)$. Data were collected at $230.0 \mathrm{~K}$. Reflections were indexed and processed, and the files scaled and corrected for absorption using APEX3 v2018. The space group was assigned and the structure was solved by direct methods using XPREP-2014/2 program and refined by full matrix least squares against $F^{2}$ with all reflections using Shelxl2018 using the graphical interface Olex2 [50]. All non-hydrogen atoms were refined with anisotropic thermal parameters, and all hydrogen atoms were included in calculated positions and refined with isotropic thermal parameters riding on those of the parent atoms. The hanging thiophene groups are highly disordered and difficult to resolve. Therefore, their electron peaks were squeezed in the refinement process. The crystallographic data for incomplete ZnBPD-4F4TS, in CIF format, have been deposited with the Cambridge Crystallographic Data Centre as CCDC 2098157. These data can be obtained free of charge from the Cambridge Crystallographic Data Centre via www.ccdc.cam.ac.uk/data_request/cif (accessed on 22 July 2021).

\subsection{Metal Ion Sensing Experiment}

ZnBPD-4F4TS crystals ( $2 \mathrm{mg}$ ) were first introduced into different metal ion aqueous solutions ( $3 \mathrm{~mL}, 500 \mathrm{ppm}$ based on metal ion) of $\mathrm{MCl}_{\mathrm{X}}\left(\mathrm{M}^{\mathrm{X}+}=\mathrm{Hg}^{2+}, \mathrm{Cd}^{2+}, \mathrm{Ni}^{2+}, \mathrm{Co}^{2+}\right.$, $\left.\mathrm{Mn}^{2+}, \mathrm{Cu}^{2+}, \mathrm{Pt}^{2+}, \mathrm{Pd}^{2+}, \mathrm{Fe}^{3+}, \mathrm{Pb}^{2+}\right), \mathrm{AgNO}_{3}$ and then heated at $80^{\circ} \mathrm{C}$ for $2 \mathrm{~h}$. After cooling to room temperature, the bulk samples were centrifuged and washed three times with water. Additionally, they were washed three times with acetone and dried under vacuum. The dried samples were then used for luminescence measurements and the luminescence data were collected. The blank sample was obtained from ZnBPD-4F4TS crystals being immersed in pure water instead of metal ion solution.

\subsection{Electrochemical Impedance Spectroscopy}

The Nyquist plots ( $Z^{\prime \prime}$ vs. $\left.Z^{\prime}\right)$ of proton-conducting MOF often show a single semicircle at high frequency, representing proton resistivity contributions of the bulk sample. The proton conductivity was deduced from the semicircle by fitting an equivalent circuit which consists of Rs, R1 and $\mathrm{W} 1$ in the frequency range from $10 \mathrm{MHz}$ to $1 \mathrm{~Hz}$. Rs corresponds to wire and electrode resistance, $\mathrm{R} 1$ is proton resistance and $\mathrm{W} 1$ is the resistivity of the grain boundary. Sometimes W1 is not necessary, because the impedance plot of the capacitive tail may not appear in the measured range due to the high magnitude of the resistivity. The water-assisted conductivities of synthesized materials were measured under different relative humidity and temperature conditions and were further fitted with different fitting circuits using the ZView software [51]. 


\section{Conclusions}

In summary, a green emitter $(x=0.264, y=0.403)$, ZnBPD-4F4TS, has been constructed from a fluorine- and thiophenethio-functionalized ligand. Exposed to various metal ions, it exhibits different reductions in luminescent intensity. In particular, the luminescence is almost fully quenched when exposed to $\mathrm{Mn}^{2+}$ ions. Moreover, $\mathrm{NH}_{4} \mathrm{Br}$-loaded $\mathbf{N H}_{4} \mathbf{B r} @ Z n B P D-4 F 4 T S$ shows a more than 25,000-fold enhanced proton conductivity compared to the original ZnBPD-4F4TS at $40{ }^{\circ} \mathrm{C}$ and $90 \% \mathrm{RH}$, serving as an example of the enhancement of proton conducting material by post-synthetic modification.

Supplementary Materials: The following are available online. Figure S1: A photograph of assynthesized ZnBPD-4F4TS single crystals. Figure S2: Solution ${ }^{1} \mathrm{H}$ NMR spectra of the activated sample of ZnBPD-4F4TS dissolved in DCl $\left(38 \%\right.$ in $\left.\mathrm{D}_{2} \mathrm{O}\right) /$ DMSO- $_{6}$ (v:v $\left.=1: 4\right)$ solution. Figure S3: Solution ${ }^{19} \mathrm{~F}$ NMR spectra of the activated sample of ZnBPD-4F4TS dissolved in DCl (38 \% in $\left.\mathrm{D}_{2} \mathrm{O}\right)$ /DMSO$d_{6}$ (v:v $=1: 4$ ) solution. Figure S4: FT-IR spectra of (a) the ligand $\mathbf{H}_{2} \mathbf{B P D}-4 \mathrm{~F} 4 \mathrm{TS}$ and (b) as-made ZnBPD-4F4TS; (c) ZnBPD-4F4TS after exposed to air for 21 days; (d) ZnBPD-4F4TS after soaking in boiling water for $30 \mathrm{~h}$. Figure S5: Thermogravimetric plots of activated ZnBPD-4F4TS. Figure S6: PXRD patterns of (a) simulation from single crystal of ZnBPD-4F4TS; (b) as-synthesized $\mathrm{NH}_{4} \mathrm{Br} @ Z n B P D-4 F 4 T S$; (c) $\mathbf{N H}_{4} \mathrm{Br} @ Z n B P D-4 F 4 T S$ after proton conduction test. Figure S7: Room temperature emission spectra of crystals $\mathbf{N H}_{4} \mathbf{B r} @ \mathbf{Z n}-4 \mathrm{~F} 4 \mathrm{TS}$ in the solid state $\left(\lambda_{\mathrm{ex}}=370 \mathrm{~nm}\right)$. Figure S8: PXRD patterns of ZnBPD-4F4TS after immersion in various metal ion solutiona. Figure S9: Proton conductivities of ZnBPD-4F4TS and NH4Br@ZnBPD-4F4TS at different temperatures (from $40{ }^{\circ} \mathrm{C}$ to $80^{\circ} \mathrm{C}$ ) and $90 \% \mathrm{RH}$. Figure S10: PXRD patterns of (a) simulation from single crystal of ZnBPD-4F4TS; (b) as-synthesized ZnBPD-4F4TS; (c) ZnBPD-4F4TS after proton conduction test. Table S1: Crystallographic refinement parameters and results of ZnBPD-4F4TS.

Author Contributions: Conceptualization, W.-M.L. and J.H.; methodology, S.-L.Z.; software, X.-H.Y.; validation, C.-M.W. and X.-H.Y.; formal analysis, W.-M.L. and J.H.; investigation, H.-Q.Z. and S.-L.Z.; data curation, H.-Q.Z. and S.-L.Z.; writing-original draft preparation, W.-M.L.; writing-review and editing, J.H.; visualization, H.-Q.Z.; supervision, J.H.; funding acquisition, W.-M.L. and J.H.; All authors have read and agreed to the published version of the manuscript.

Funding: This work was funded by the National Natural Science Foundation of China (21871061 and 21901046), the Foundation of Basic and Applied Basic Research of Guangdong Province (2020B1515120024 and 2021A1515010274), Local Innovative and Research Teams Project of Guangdong Pearl River Talents Program (2017BT01Z032), Science and Technology Planning Project of Guangdong Province (2021A0505030066) and Science and Technology Program of Guangzhou (201807010026).

Institutional Review Board Statement: Not applicable.

Informed Consent Statement: Not applicable.

Data Availability Statement: All data are included in the article.

Conflicts of Interest: The authors declare no conflict of interest.

Sample Availability: Samples of the compounds are not available from the authors.

\section{References}

1. Yan, B. Luminescence response mode and chemical sensing mechanism for lanthanide-functionalized metal-organic framework hybrids. Inorg. Chem. Front. 2021, 8, 201-233. [CrossRef]

2. Wagner, M.; Lin, K.Y.A.; Oh, W.D.; Lisak, G. Metal-organic frameworks for pesticidal persistent organic pollutants detection and adsorption-A mini review. J. Hazard. Mater. 2021, 413, 125325. [CrossRef] [PubMed]

3. Yi, F.-Y.; Chen, D.; Wu, M.-K.; Han, L.; Jiang, H.-L. Chemical Sensors Based on Metal-Organic Frameworks. ChemPlusChem 2016, 81, 675-690. [CrossRef] [PubMed]

4. Pan, M.; Liao, W.M.; Yin, S.Y.; Sun, S.S.; Su, C.Y. Single-Phase White-Light-Emitting and Photoluminescent Color-Tuning Coordination Assemblies. Chem. Rev. 2018, 118, 8889-8935. [CrossRef]

5. Yan, B. Lanthanide-Functionalized Metal-Organic Framework Hybrid Systems To Create Multiple Luminescent Centers for Chemical Sensing. Acc. Chem. Res. 2017, 50, 2789-2798. [CrossRef]

6. Feng, L.; Pang, J.; She, P.; Li, J.L.; Qin, J.S.; Du, D.Y.; Zhou, H.C. Metal-Organic Frameworks Based on Group 3 and 4 Metals. Adv. Mater. 2020, 32, 2004414. [CrossRef] 
7. Sun, T.; Gao, Y.; Du, Y.; Zhou, L.; Chen, X. Recent Advances in Developing Lanthanide Metal-Organic Frameworks for Ratiometric Fluorescent Sensing. Front. Chem. 2020, 8, 624592. [CrossRef] [PubMed]

8. Mahata, P.; Mondal, S.K.; Singha, D.K.; Majee, P. Luminescent rare-earth-based MOFs as optical sensors. Dalton Trans. 2017, 46, 301-328. [CrossRef]

9. Lai, C.; Wang, Z.; Qin, L.; Fu, Y.; Li, B.; Zhang, M.; Liu, S.; Li, L.; Yi, H.; Liu, X.; et al. Metal-organic frameworks as burgeoning materials for the capture and sensing of indoor VOCs and radon gases. Coord. Chem. Rev. 2021, 427, 213565. [CrossRef]

10. Rasheed, T.; Nabeel, F. Luminescent metal-organic frameworks as potential sensory materials for various environmental toxic agents. Coord. Chem. Rev. 2019, 401, 213065. [CrossRef]

11. Zhang, Y.; Yuan, S.; Day, G.; Wang, X.; Yang, X.; Zhou, H.-C. Luminescent sensors based on metal-organic frameworks. Coord. Chem. Rev. 2018, 354, 28-45. [CrossRef]

12. Cao, Y.; Zhang, Y.; Gu, L.-W.; Qin, X.-M.; Li, H.-Y.; Bian, H.-D.; Huang, F.-P. A zinc ${ }^{2+}$-dpbt framework: Luminescence sensing of $\mathrm{Cu}^{2+}, \mathrm{Ag}^{+}, \mathrm{MnO}_{4}{ }^{-}$and $\mathrm{Cr}(\mathrm{VI})\left(\mathrm{Cr}_{2} \mathrm{O}_{7}{ }^{2-}\right.$ and $\left.\mathrm{CrO}_{4}{ }^{2-}\right)$ ions. New J. Chem. 2020, 44, 10681-10688. [CrossRef]

13. Gu, T.Y.; Dai, M.; Young, D.J.; Ren, Z.G.; Lang, J.P. Luminescent Zn(II) Coordination Polymers for Highly Selective Sensing of $\mathrm{Cr}(\mathrm{III})$ and $\mathrm{Cr}(\mathrm{VI})$ in Water. Inorg. Chem. 2017, 56, 4669-4679.

14. Lv, R.; Wang, J.; Zhang, Y.; Li, H.; Yang, L.; Liao, S.; Gu, W.; Liu, X. An amino-decorated dual-functional metal-organic framework for highly selective sensing of $\mathrm{Cr}(\mathrm{III})$ and $\mathrm{Cr}(\mathrm{VI})$ ions and detection of nitroaromatic explosives. J. Mater. Chem. A 2016, 4, 15494-15500. [CrossRef]

15. Wu, H.; Yang, J.; Liu, Y.-Y.; Ma, J.-F. pH-Controlled Assembly of Two Unusual Entangled Motifs Based on a Tridentate Ligand and Octamolybdate Clusters: 1D $+1 \mathrm{D} \rightarrow 3 \mathrm{D}$ Poly-Pseudorotaxane and 2D $\rightarrow 2 \mathrm{D} \rightarrow 3 \mathrm{D}$ Polycatenation. Cryst. Growth Des. 2012, 12, 2272-2276. [CrossRef]

16. Xiang, H.; Gao, W.-Y.; Zhong, D.-C.; Jiang, L.; Lu, T.-B. The diverse structures of Cd(II) coordination polymers with 1,3,5benzenetribenzoate tuned by organic bases. CrystEngComm 2011, 13, 5825-5832. [CrossRef]

17. Razavi, S.A.A.; Morsali, A. Metal ion detection using luminescent-MOFs: Principles, strategies and roadmap. Coord. Chem. Rev. 2020, 415, 213299. [CrossRef]

18. Kobielska, P.A.; Howarth, A.J.; Farha, O.K.; Nayak, S. Metal-organic frameworks for heavy metal removal from water. Coord. Chem. Rev. 2018, 358, 92-107. [CrossRef]

19. Li, Y.X.; Li, S.J.; Yan, P.F.; Wang, X.Y.; Yao, X.; An, G.H.; Li, G.M. Luminescence-colour-changing sensing of Mn(2+) and Ag(+) ions based on a white-light-emitting lanthanide coordination polymer. Chem. Commun. 2017, 53, 5067-5070. [CrossRef] [PubMed]

20. Wu, Y.; Wu, J.; Luo, Z.; Wang, J.; Li, Y.; Han, Y.; Liu, J. Fluorescence detection of $\mathrm{Mn}^{2+}, \mathrm{Cr}_{2} \mathrm{O}_{7}^{2-}$ and nitroexplosives and photocatalytic degradation of methyl violet and rhodamine B based on two stable metal-organic frameworks. RSC Adv. 2017, 7, 10415-10423. [CrossRef]

21. Xie, X.-X.; Yang, Y.-C.; Dou, B.-H.; Li, Z.-F.; Li, G. Proton conductive carboxylate-based metal-organic frameworks. Coord. Chem. Rev. 2020, 403, 213100. [CrossRef]

22. Lim, D.W.; Kitagawa, H. Proton Transport in Metal-Organic Frameworks. Chem. Rev. 2020, 120, 8416-8467. [CrossRef]

23. Li, W.-H.; Deng, W.-H.; Wang, G.-E.; Xu, G. Conductive MOFs. EnergyChem 2020, 2, 100029. [CrossRef]

24. Deng, X.; Hu, J.-Y.; Luo, J.; Liao, W.-M.; He, J. Conductive Metal-Organic Frameworks: Mechanisms, Design Strategies and Recent Advances. Top. Curr. Chem. 2020, 378, 27. [CrossRef]

25. Bao, S.-S.; Shimizu, G.K.H.; Zheng, L.-M. Proton conductive metal phosphonate frameworks. Coord. Chem. Rev. 2019, 378, 577-594. [CrossRef]

26. Zhang, X.; Wang, B.; Alsalme, A.; Xiang, S.; Zhang, Z.; Chen, B. Design and applications of water-stable metal-organic frameworks: Status and challenges. Coord. Chem. Rev. 2020, 423, 213507. [CrossRef]

27. Yuan, S.; Feng, L.; Wang, K.; Pang, J.; Bosch, M.; Lollar, C.; Sun, Y.; Qin, J.; Yang, X.; Zhang, P.; et al. Stable Metal-Organic Frameworks: Design, Synthesis, and Applications. Adv. Mater. 2018, 30, 1704303. [CrossRef] [PubMed]

28. Wang, C.; Liu, X.; Keser Demir, N.; Chen, J.P.; Li, K. Applications of water stable metal-organic frameworks. Chem. Soc. Rev. 2016, 45, 5107-5134. [CrossRef] [PubMed]

29. Mileo, P.G.M.; Adil, K.; Davis, L.; Cadiau, A.; Belmabkhout, Y.; Aggarwal, H.; Maurin, G.; Eddaoudi, M.; Devautour-Vinot, S. Achieving Superprotonic Conduction with a 2D Fluorinated Metal-Organic Framework. J. Am. Chem. Soc. 2018, 140, 13156-13160. [CrossRef] [PubMed]

30. Pili, S.; Argent, S.P.; Morris, C.G.; Rought, P.; Garcia-Sakai, V.; Silverwood, I.P.; Easun, T.L.; Li, M.; Warren, M.R.; Murray, C.A.; et al. Proton Conduction in a Phosphonate-Based Metal-Organic Framework Mediated by Intrinsic "Free Diffusion inside a Sphere". J. Am. Chem. Soc. 2016, 138, 6352-6355. [CrossRef] [PubMed]

31. Taylor, J.M.; Dawson, K.W.; Shimizu, G.K.H. A Water-Stable Metal-Organic Framework with Highly Acidic Pores for ProtonConducting Applications. J. Am. Chem. Soc. 2013, 135, 1193-1196. [CrossRef]

32. Horike, S.; Umeyama, D.; Inukai, M.; Itakura, T.; Kitagawa, S. Coordination-network-based ionic plastic crystal for anhydrous proton conductivity. J. Am. Chem. Soc. 2012, 134, 7612-7615. [CrossRef]

33. Panda, T.; Kundu, T.; Banerjee, R. Structural isomerism leading to variable proton conductivity in indium(III) isophthalic acid based frameworks. Chem. Commun. 2013, 49, 6197-6199. [CrossRef]

34. Xing, X.S.; Fu, Z.H.; Zhang, N.N.; Yu, X.Q.; Wang, M.S.; Guo, G.C. High proton conduction in an excellent water-stable gadolinium metal-organic framework. Chem. Commun. 2019, 55, 1241-1244. [CrossRef] 
35. Liu, L.; Yao, Z.; Ye, Y.; Liu, C.; Lin, Q.; Chen, S.; Xiang, S.; Zhang, Z. Enhancement of Intrinsic Proton Conductivity and Aniline Sensitivity by Introducing Dye Molecules into the MOF Channel. ACS Appl. Mater. Interfaces 2019, 11, 16490-16495. [CrossRef]

36. Ye, Y.; Guo, W.; Wang, L.; Li, Z.; Song, Z.; Chen, J.; Zhang, Z.; Xiang, S.; Chen, B. Straightforward Loading of Imidazole Molecules into Metal-Organic Framework for High Proton Conduction. J. Am. Chem. Soc. 2017, 139, 15604-15607. [CrossRef]

37. Phang, W.J.; Jo, H.; Lee, W.R.; Song, J.H.; Yoo, K.; Kim, B.; Hong, C.S. Superprotonic conductivity of a UiO-66 framework functionalized with sulfonic acid groups by facile postsynthetic oxidation. Angew. Chem. Int. Ed. 2015, 54, 5142-5146. [CrossRef] [PubMed]

38. Li, X.; Sun, X.; Li, X.; Fu, Z.; Su, Y.; Xu, G. Porous Cadmium(II) Anionic Metal-Organic Frameworks Based on Aromatic Tricarboxylate Ligands: Encapsulation of Protonated Flexible Bis(2-methylimidazolyl) Ligands and Proton Conductivity. Cryst. Growth Des. 2015, 15, 4543-4548. [CrossRef]

39. Zhou, H.Q.; He, Y.; Hu, J.Y.; Chung, L.H.; Gu, Q.; Liao, W.M.; Zeller, M.; Xu, Z.; He, J. Conjugated crosslinks boost the conductivity and stability of a single crystalline metal-organic framework. Chem. Commun. 2021, 57, 187-190. [CrossRef] [PubMed]

40. Eddaoudi, M.; Kim, J.; Rosi, N.; Vodak, D.; Wachter, J.; O’Keeffe, M.; Yaghi, O.M. Systematic design of pore size and functionality in isoreticular MOFs and their application in methane storage. Science 2002, 295, 469-472. [CrossRef] [PubMed]

41. Dong, L.Z.; Zhang, L.; Liu, J.; Huang, Q.; Lu, M.; Ji, W.X.; Lan, Y.Q. Stable Heterometallic Cluster-Based Organic Framework Catalysts for Artificial Photosynthesis. Angew. Chem. Int. Ed. 2020, 59, 2659-2663. [CrossRef] [PubMed]

42. Chen, J.; Li, B.; Shi, Z.; He, C.; Duan, C.; Zhang, T.; Wang, L.-Y. Crystal engineering of coordination-polymer-based iodine adsorbents using a $\pi$-electron-rich polycarboxylate aryl ether ligand. CrystEngComm 2020, 22, 6612-6619. [CrossRef]

43. Cui, J.; Li, Y.; Guo, Z.; Zheng, H. A porous metal-organic framework based on Zn6O2 clusters: Chemical stability, gas adsorption properties and solvatochromic behavior. Chem. Commun. 2013, 49, 555-557. [CrossRef] [PubMed]

44. Lou, X.-H.; Xu, C.; Li, H.-M.; Wang, Z.-Q.; Guo, H.; Xue, D.-X. Metal-directed topological diversity of three fluorescent metalorganic frameworks based on a new tetracarboxylate strut. CrystEngComm 2013, 15, 4606-4610. [CrossRef]

45. Ma, D.-Y.; Qin, L.; Lei, J.-M.; Liang, Y.-Q.; Lin, W.-J.; Yan, J.-J.; Ding, W.-Q.; Guo, H.-F.; Ling, Y. Structural diversity of a series of coordination polymers built from 5-substituted isophthalic acid with or without a methyl-functionalized N-donor ligand. CrystEngComm 2016, 18, 1363-1375. [CrossRef]

46. Li, X.-M.; Dong, L.-Z.; Li, S.-L.; Xu, G.; Liu, J.; Zhang, F.-M.; Lu, L.-S.; Lan, Y.-Q. Synergistic Conductivity Effect in a Proton Sources-Coupled Metal-Organic Framework. ACS Energy Lette. 2017, 2, 2313-2318. [CrossRef]

47. Li, Y.-Z.; Fu, Z.-H.; Xu, G. Metal-organic framework nanosheets: Preparation and applications. Coord. Chem. Rev. 2019, 388, 79-106. [CrossRef]

48. Kreuer, K.-D. Proton Conductivity: Materials and Applications. Chem. Mater. 1996, 8, 610-641. [CrossRef]

49. Kreuer, K.-D.; Rabenau, A.; Weppner, W. Vehicle Mechanism, A New Model for the Interpretation of the Conductivity of Fast Proton Conductors. Angew. Chem. Int. Ed. 1982, 21, 208-209. [CrossRef]

50. Dolomanov, O.V.; Bourhis, L.J.; Gildea, R.J.; Howard, J.A.K.; Puschmann, H. OLEX2: A complete structure solution, refinement and analysis program. J. Appl. Crystallogr. 2009, 42, 339-341. [CrossRef]

51. Vladikova, D. Selectivity study of the differential impedance analysis-Comparison with the complex non-linear least-squares method. Electrochim. Acta 2002, 47, 2943-2951. [CrossRef] 